\title{
Making and viewing stereoscopic surgical videos with smartphones and virtual reality headset
}

Eye (2016) 30, 503-504; doi:10.1038/eye.2015.282; published online 22 January 2016

Smartphones have been used in anterior and posterior segment photography ${ }^{1,2}$ and adaptors are available to enable the recording of surgical videos. The two-dimensional (2D) images can be of excellent quality but are lacking when compared to the stereoscopic three-dimensional (3D) view through the slit lamp or microscope eyepieces.

A number of companies (eg Google/Samsung/ Zeiss) are using smartphones and head-mounted Virtual Reality (VR) headsets (eg Google Cardboard/Samsung Gear VR/Zeiss VR One) for the purpose of immersive $3 \mathrm{D}$ video gaming or movie viewing. These are effectively headmounted stereoscopes into which a smart phone is placed to display the image. Using two smartphones and a VR headset we have developed a way of viewing slit lamp and surgical videos in 3D.

We connected two iPhone 4 Ss to the slit lamp or operating microscope using 3D-printed eyepiece adaptors (Figure 1). Video of signs such as anterior chamber cells in anterior uveitis, and of cataract and strabismus surgery were recorded from left and right eyepieces. The two videos were synchronized and displayed sideby-side (left video on the left and the right video on the right) in a single video using videoediting software.

This side-by-side video is loaded onto the iPhone. It is then viewed in stereoscopic 3D using an inexpensive VR headset into which the phone is placed (Figure 2, Supplementary video).

Recently, novel methods have been used to record video of eye surgery, including Google Glass $^{3}$ and two GoPro cameras to create a stereoscopic video. ${ }^{4}$ These methods are limited by the wide field of view, meaning the object of interest is quite small. This limits the capability of such methods to create a useful surgical educational video. The image quality with our technique is excellent with a good stereoscopic effect. Ophthalmic trainees can view surgery in the same way as when looking through the microscope assistant eyepieces. This allows a much more immersive and instructional view

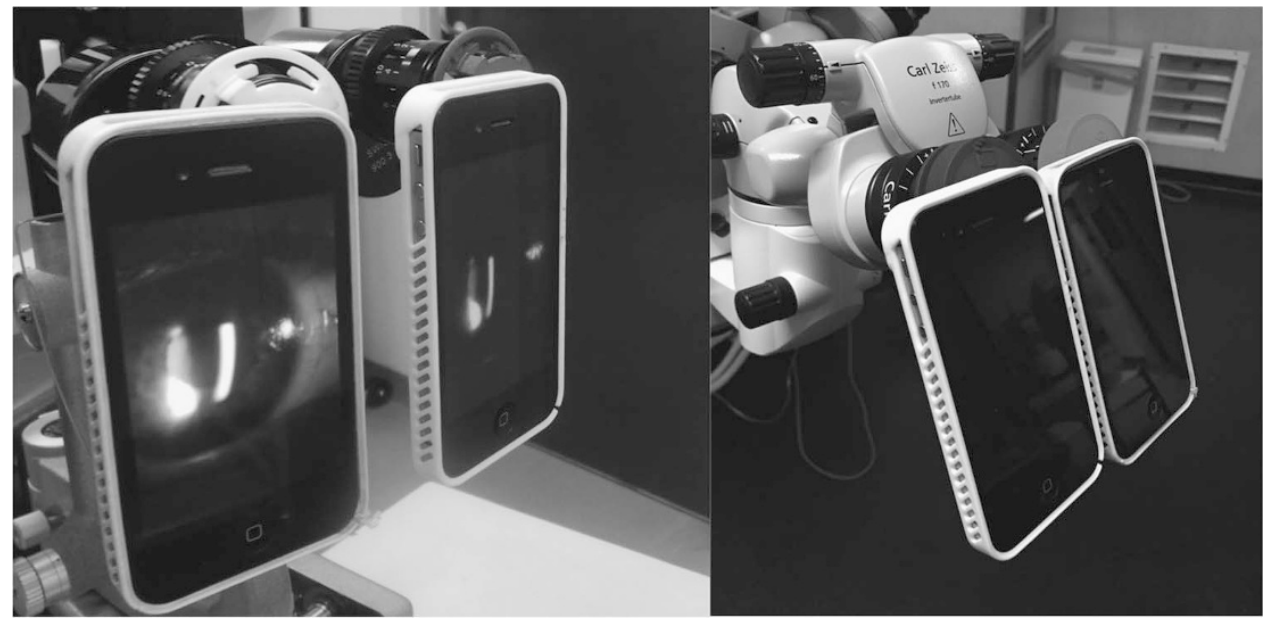

Figure 1 Two phones attached to the slit lamp or microscope eyepieces record images from two perspectives to create a stereoscopic effect. 


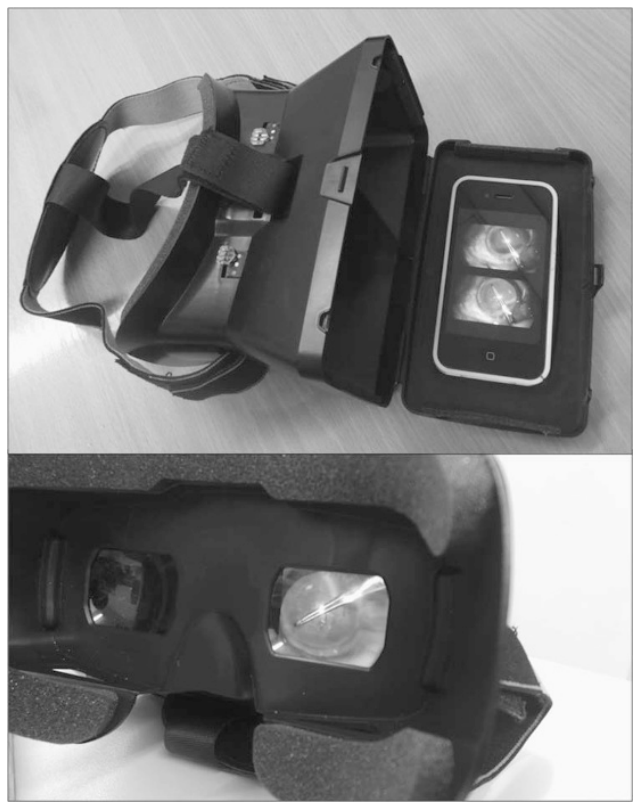

Figure 2 iPhone loaded into the VR headset for viewing of video with stereoscopic 3D effect.

of surgery than is available with $2 \mathrm{D}$ surgical educational videos. In future, surgical trainees worldwide could view an online library of 3D instructional videos using a VR headset as if they were in theatre with the surgeon.

\section{Conflict of interest}

The authors declare no conflict of interest.

\section{References}

1 Barsam A, Bhogal M, Morris S, Little B. Anterior segment slit lamp photography using the iPhone. J Cataract Refract Surg 2010; 36(7): 1240-1241.

2 Haddock LJ, Kim DY, Mukai S. Simple, inexpensive technique for high-quality smartphone fundus photography in human and animal eyes. J Ophthalmol 2013; 2013: 518479.

3 Rahimy E, Garg SJ. Google glass for recording Scleral Buckling Surgery. JAMA Ophthalmol 2015; 133(6): 710-711.

4 Birnbaum FA, Wang A, Brady CJ. Stereoscopic surgical recording using GoPro cameras: a low-cost means for capturing external eye surgery. JAMA Ophthalmol 2015; 133(12): 1483-1484.

K Gallagher ${ }^{1,2}$, S Jain ${ }^{1}$ and N Okhravi $i^{2,3,4}$

${ }^{1}$ Department of Ophthalmology, Royal Free London Hospital, London, UK

${ }^{2}$ Department of Undergraduate Education, Moorfields Eye Hospital NHSFT, London, UK

${ }^{3}$ NIHR Biomedical Research Centre at Moorfields Eye Hospital NHSFT and UCL Institute of Ophthalmology,

London, UK

${ }^{4}$ Moorfields Eye Hospital NHSFT, London, UK

Correspondence: K Gallagher, Department of Ophthalmology, Royal Free London Hospital, Pond Street, London NW3 2QG, UK Tel: +44 (0)20 7794 0500; Fax: +44 (0)20 77940501. E-mail: gallagherkr@gmail.com

Supplementary Information accompanies this paper on Eye website (http://www.nature.com/eye) 